DOI: $10.15593 / 2224-9982 / 2017.51 .12$

УДК 620.17

\author{
М.Ш. Нихамкин, Д.Г. Соломонов
}

Пермский национальный исследовательский политехнический университет, Пермь, Россия

\author{
ПРИМЕНЕНИЕ ЭКСПЕРИМЕНТАЛЬНОГО МОДАЛЬНОГО АНАЛИЗА \\ ДЛЯ ИДЕНТИФИКАЦИИ ПАРАМЕТРОВ МОДЕЛИ СЛОИСТОГО \\ УГЛЕПЛАСТИКА
}

\begin{abstract}
Расчет модальных характеристик (собственных частот и форм), необходимых для того, чтобы исключить резонансные колебания изделий из полимерных композиционных материалов (ПКМ), требует надежных данных о механических характеристиках материала. Проблема состоит в большом (по сравнению с изотропными материалами) количестве характеристик упругости, а также в том, что эти параметры зависят от широкого круга структурных и технологических факторов. Данные о характеристиках материалов, приведенные в литературе, зачастую противоречивы и при проведении ответственных расчетов требуют дополнительной проверки.

Цель настоящей работы - разработка методики идентификации параметров модели упругого поведения ПКМ по результатам экспериментального модального анализа. Объект исследования - слоистый углепластик на основе равнопрочной углеродной ткани, широко применяемый в авиастроении.

Для экспериментального определения собственных частот и фрорм колебаний используется метод сканирующей лазерной виброметрии, для расчетного модального анализа - метод конечных элементов. Модель материала - слоистый композит с ортотропными линейно упругими слоями. Задача идентификации параметров модели материала рассматривается как задача минимизации расхождения расчетных собственных частот с экспериментальными. Для ее решения используется метод квазислучайного поиска.

Разработанная методика может быть рекомендована для определения параметров модели материала, необходимых для расчетов модальных характеристик изделий из ПКМ.

Ключевые слова: полимерные композиционные материалы, механические характеристики, модальный анализ, метод конечных элементов.
\end{abstract}

\author{
M.Sh. Nikhamkin, D.G. Solomonov
}

Perm National Research Polytechnic University, Perm, Russian Federation

\title{
APPLICATION OF EXPERIMENTAL MODAL ANALYSIS TO IDENTIFY THE PARAMETERS OF THE MODEL OF LAMINATED CARBON FIBER REINFORCED PLASTIC
}

The calculation of modal characteristics (natural frequencies and vibration modes) of products made of polymer composite materials, necessary to exclude their resonance oscillations, requires reliable data on the mechanical characteristics of the material. The problem is a large (in comparison with isotropic materials) quantity of elasticity characteristics, and also in the fact that these parameters depend on a wide range of structural and technological factors. Data on the characteristics of materials, given in the literature, are often contradictory and require additional verification when carrying out responsible calculations.

The purpose of this work is to develop a methodology for identifying models of elastic behavior of polymeric composite materials based on the results of experimental modal analysis using the example of laminated carbon fiber reinforced plastic. The object of investigation is a layered carbon fiber reinforced plastic based on an equally strong carbon cloth widely used in aircraft building.

The method of scanning laser vibrometry is used for experimental determination of natural frequencies and modes of oscillations. For the numerical modal analysis, the finite element method is used. The material model is a layered composite with orthotropic linearly elastic layers. The task of identifying the material model is considered as the problem of minimizing the discrepancy between the calculated natural frequencies and the experimental ones. To solve it, the quasi-random search method is used.

The developed method can be recommended for determination of parameters of material models for calculating modal characteristics of polymer composite products.

Keywords: laminated carbon fiber reinforced plastic, mechanical characteristics, modal analysis, finite element method. 


\section{Введение}

Полимерные композиционные материалы (ПКМ), в частности углепластики, широко используются для изготовления нагруженных деталей самолетов и авиационных двигателей благодаря возможности облегчения конструкции без снижения прочности [1-4]. Известно, например, что использование ПКМ в конструкции планера самолетов Boeing 787 достигает $50 \%$ по весу $[2,4]$. Широкое применение ПКМ предусматривается при создании отечественных самолетов и авиационных двигателей нового поколения $[2,3]$.

Одна из проблем применения ПКМ в авиастроении - обеспечение прочности в условиях вибраций, характерных для авиационной техники. При значительных амплитудах колебаний, которые могут возникнуть, например, в условиях резонанса, может произойти разрушение элементов конструкции из ПКМ, ведущее к опасным последствиям. Чтобы исключить резонансные колебания, надо еще на стадии проектирования рассчитывать модальные характеристики деталей: их собственные частоты и формы колебаний. В случае изделий из ПКМ такой расчет осложняется отсутствием надежных данных о механических характеристиках. Проблема состоит в большом (по сравнению с изотропными материалами) количестве параметров, входящих в модель материала, а также в том, что эти параметры зависят от материалов волокон и связующего, схемы армирования, технологических факторов [5, 6]. Так, например, модель ортотропного упругого материала содержит девять параметров. Их определение - трудоемкая экспериментальная задача. Данные о характеристиках материалов, приведенные в литературе, зачастую противоречивы и при проведении ответственных расчетов требуют дополнительной проверки.

Для расчетного анализа напряженно-деформированного состояния и модального анализа конструкций, в том числе из ПКМ, эффективно используется метод конечных элементов (МКЭ) (см, например, работы [7-12]). Из-за неопределенностей и допущений, неизбежных при моделировании сложных конструкций, корректное применение МКЭ требует проведения верификации, а при необходимости - идентификации (настройки) конечно-элементных (КЭ) моделей по экспериментальным данным. Под идентификацией КЭ-модели понимается такое изменение ее параметров, которое сводит к минимуму отличия между расчетными и экспериментальными данными. В ряде работ последних лет [11-17] для этого используются результаты экспериментального модального анализа.

В работе [12] корректировка КЭ-модели рабочего колеса турбины проводилась за счет подбора условных значений плотности материала и модуля Юнга, обеспечивающего минимальное расхождение расчетных и экспериментальных значений собственных частот. Коррекция проводилась с помощью коммерческой программы LMS Virtual.Lab Optimization. В работах $[13,14]$ параметрами, за счет которых выполняется настройка КЭ-модели по собственным частотам, были условные жесткости отдельных конечных элементов. В работах $[15,16]$ идентификация КЭ-моделей рассматривается как задача многокритериальной многопараметрической оптимизации. Для ее решения предлагается алгоритм, основанный на анализе чувствительности. В работе [17] результаты экспериментального модального анализа использовались для оценки эффективных характеристик свойств сотового заполнителя. Анализ публикаций по проблеме идентификации КЭ-моделей по данным экспериментального модального анализа показывает, что опыт идентификации КЭ-моделей применительно к конструкциям из ПКМ недостаточен.

Цель настоящей работы - разработка методики идентификации параметров модели упругого поведения ПКМ по результатам экспериментального модального анализа на примере слоистого углепластика.

\section{Объект исследования}

Объект исследования - слоистый углепластик ВКУ-39 на основе равнопрочной углеродной ткани Porsher 3692 и связующего ВСЭ-1212, широко применяемый в авиастроении [3]. Исследование проводилось на образцах, представляющих собой пластину длиной 250 мм, шири- 
ной 24,6 мм и толщиной 4,2 мм (рис. 1). Подобные образцы рекомендованы стандартом ASTM для определения механических характеристик ПКМ при растяжении и усталостных испытаниях $[18,19]$. Образец состоит из 20 слоев углепрепрега 0,21 мм со схемой армирования $(90 / 45 / 0 /-45 / 0) * 4$. Масса образца 40 г.

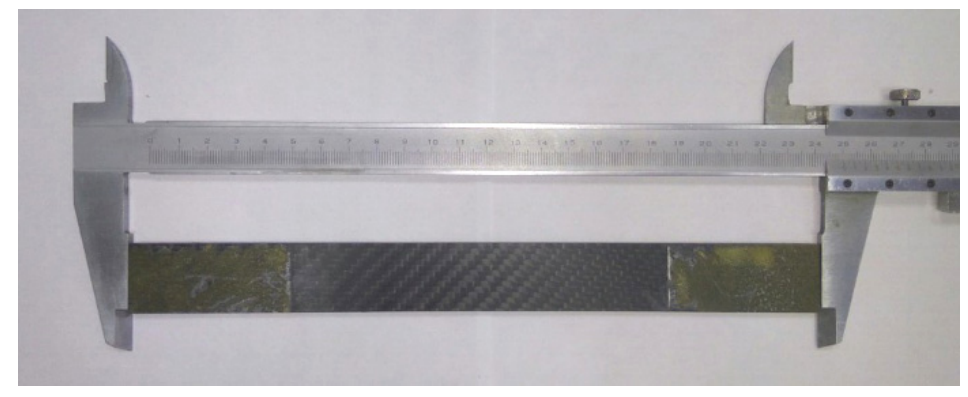

Рис. 1. Образец - пластина из углепластика

\section{Методика и результаты экспериментального модального анализа}

Задача экспериментального модального анализа (ЭМА) состояла в получении данных о собственных частотах и формах колебаний образца, необходимых для последующей идентификации расчетной модели. Современные средства ЭМА, в частности используемый в настоящей работе метод сканирующей лазерной виброметрии, позволяют не только получать с высокой точностью данные о собственных частотах, но и определять с высоким пространственным разрешением собственные формы колебаний.

Методика ЭМА основана на представлении исследуемого объекта как колебательной системы с конечным числом $n$ степеней свободы. Экспериментальное определение собственных частот системы и соответствующих собственных форм базируется на анализе матрицы передаточных функций $[H]$, каждый элемент которой представляет собой результат измерений отдельной частотной характеристики как отношения [20, 21]

$$
H_{i j}(\omega)=X_{i}(\omega) / F_{j}(\omega), \quad i, j=1, \ldots, n,
$$

где $X_{i}(\omega)$ - частотная функция реакции в виде скорости или ускорения для $i$-й степени свободы $(\mathrm{CC})$ на воздействие $F_{j}(\omega)$, соответствующее $j$-й $\mathrm{CC} ; \omega$ - частота.

При сканирующей лазерной виброметрии к одной СС объекта $i$ прикладывается воздействие в виде внешней силы, а реакция в виде виброскорости измеряется во множестве СС $j=1, \ldots, n$ при их последовательным сканировании [21-24]. Далее по соотношению (1) определяются компоненты матрицы передаточных функций $H_{i j}(\omega)$. Собственные частоты определяются по пикам на замеренной амплитудно-частотной характеристике (АЧХ) $H_{i j}(\omega)$. Для определения собственной формы колебаний одна из степеней свободы принимается опорной и проводится серия измерений амплитуд виброскорости для всех СС при соответствующем значении модальной частоты. Число степеней свободы $n$ при ЭМА выбирается так, чтобы обеспечить достаточное пространственное разрешение для надежного представления форм колебаний.

Принципиальное достоинство метода сканирующей лазерной вибромерии - бесконтактное измерение вибраций, благодаря чему колебательная система не искажается введением в нее дополнительных масс датчиков и жесткости кабелей, препарировка объекта датчиками не требуется. Другое достоинство - высокая пространственная разрешающая способность: плотность точек измерения отклика ограничена только точностью фокусировки лазера [21].

При проведении экспериментального модального анализа исследуемого образца использовалась методика, основанная на применении трехкомпонентного сканирующего лазерного виброметра Polytec PSV400-3D [23-25]. Он представляет собой исследовательский лазерноцифровой измерительный комплекс, измерение виброскорости в котором основано на исполь- 
зовании эффекта Доплера. PSV400-3D состоит из трех оптических сканирующих лазерных головок, модуля сканирования геометрии и системы управления на базе промышленного компьютера.

Исследуемый образец закреплялся на жесткой металлической раме в податливых упругих подвесах (рис. 2). Такая схема, часто используемая в экспериментальном модальном анализе, позволяет максимально приблизить условия закрепления к отсутствию ограничений на перемещения («свободный подвес»), удобному для воспроизведения в последующих расчетах.

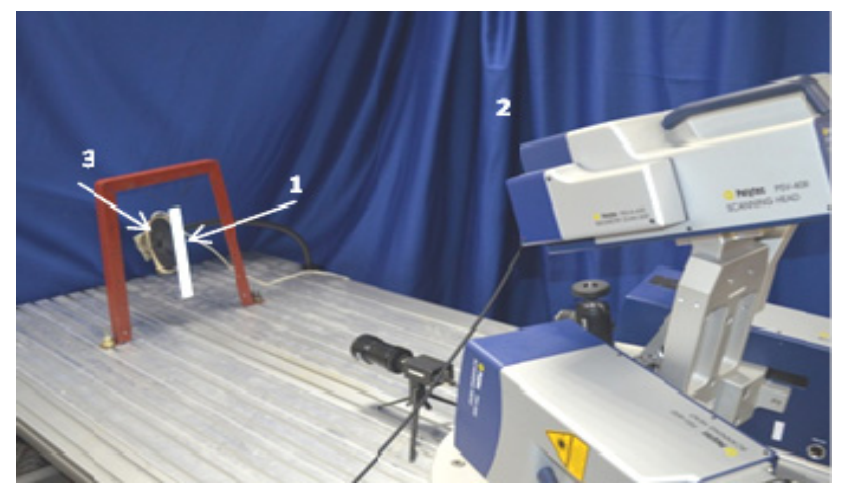

Рис. 2. Экспериментальная установка: 1 - образец; 2 - лазерный виброметр; 3 - акустический возбудитель колебаний

Возбуждение колебаний осуществлялось с помощью акустического динамика. На него подавался сигнал, изменяющийся во времени по гармоническому закону с постоянной амплитудой и возрастающей до 6400 Гц частотой (Periodic Chirp). Проведено пять экспериментов с различными параметрами сетки сканирования, которая включала в себя от 25 до 165 узлов.

На рис. 3 приведена в качестве примера АЧХ образца. В исследованном диапазоне частот выявлено восемь собственных форм колебаний. В табл. 1 приведены значения собственных частот колебаний, полученные в пяти экспериментах. Рассеяние экспериментальных данных в табл. 1 оценивается значениями коэффициента вариации, который лежит в пределах 0,57 \%, что свидетельствует о высокой точности экспериментального определения собственных частот.

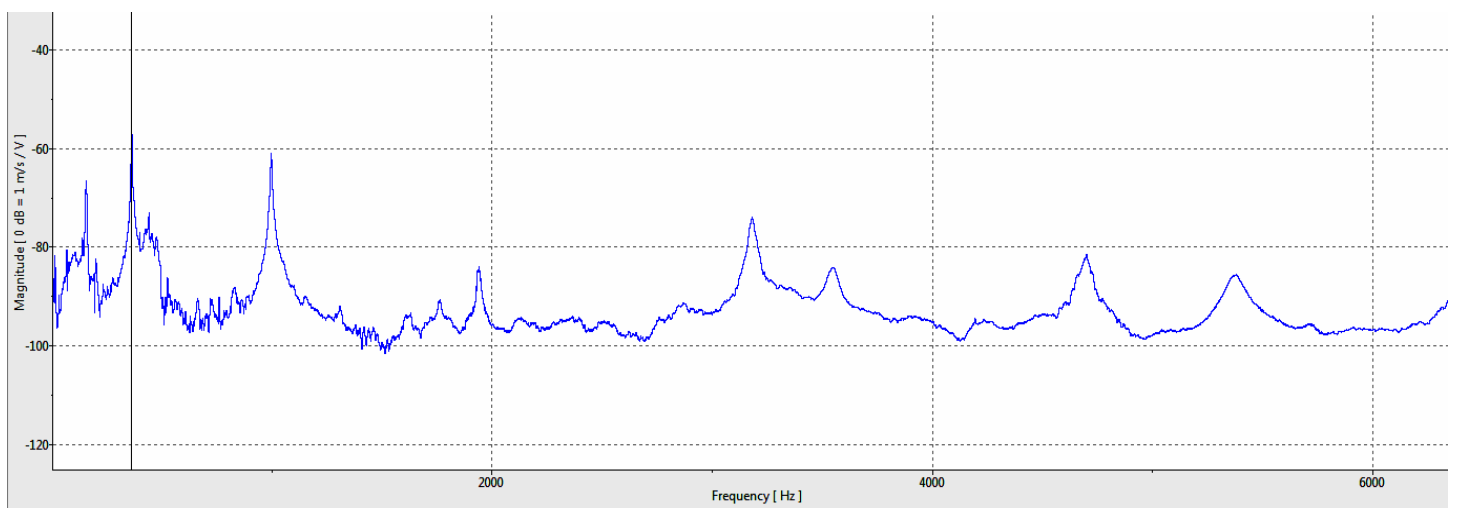

Рис. 3. Пример АЧХ образца

Таблица 1

Результаты определения собственных частот колебаний, Гц

\begin{tabular}{|c|c|c|c|c|c|c|c|}
\hline \multirow{2}{*}{$\begin{array}{c}\text { № } \\
\text { п/п }\end{array}$} & \multicolumn{3}{|c|}{ Номер эксперимента / число точек сканирования } & \multirow{2}{*}{$\begin{array}{c}\text { Среднее } \\
\text { значение }\end{array}$} & $\begin{array}{c}\text { Коэффициент } \\
\text { вариции, \% }\end{array}$ \\
\hline 1 & $1 / 25$ & $2 / 51$ & $3 / 51$ & $4 / 165$ & $5 / 165$ & 363,4 & 0,571 \\
\hline 2 & 363 & 364 & 360 & 365 & 365 & 363,4 & 0,135 \\
\hline 3 & 1765 & 998 & 998 & 998 & 998 & 997,4 & 0,111 \\
\hline 4 & 1935 & 1765 & 1764 & 1762 & 1762 & 1762,6 & 0,180 \\
\hline 5 & 3178 & 3181 & 3177 & 3181 & 3181 & 3179,6 & 0,061 \\
\hline 6 & 3540 & 3551 & 3556 & 3548 & 3549 & 3548,8 & 0,164 \\
\hline 7 & 4694 & 4702 & 4690 & 4688 & 4700 & 4694,8 & 0,130 \\
\hline 8 & 5342 & 5378 & 5371 & 5374 & 5381 & 5369,2 & 0,292 \\
\hline
\end{tabular}


Собственные формы колебаний приведены в табл. 2. Среди них пять изгибных и три крутильных формы. Эти собственные формы используются для последующего сравнения с расчетными при оценке точности расчетов.

Таблица 2

Собственные частоты и формы колебаний пластины: эксперимент и расчет

\begin{tabular}{|c|c|c|c|c|c|}
\hline \multirow{2}{*}{$\begin{array}{l}\text { № } \\
\text { п/п }\end{array}$} & \multicolumn{2}{|c|}{ Эксперимент } & \multicolumn{2}{|c|}{ Расчет МКЭ } & \multirow{2}{*}{$\begin{array}{c}\text { Расхож- } \\
\text { дение, } \\
\%\end{array}$} \\
\hline & $\begin{array}{c}\text { Собственная форма } \\
\text { колебаний }\end{array}$ & $\begin{array}{c}\text { Собственная } \\
\text { частота, Гц }\end{array}$ & $\begin{array}{c}\text { Собственная форма } \\
\text { колебаний }\end{array}$ & $\begin{array}{c}\text { Собственная } \\
\text { частота, Гц }\end{array}$ & \\
\hline 1-я изгибная & & 363 & & 389,0 & 7,05 \\
\hline 2-я изгибная & & 997 & & 1063,2 & 6,60 \\
\hline $\begin{array}{l}1-я \\
\text { крутильная }\end{array}$ & & 1763 & & 1744,7 & $-1,02$ \\
\hline 3-я изгибная & & 1940 & & 2057,4 & 6,01 \\
\hline 4-я изгибная & & 3180 & & 3342,9 & 5,14 \\
\hline $\begin{array}{l}\text { 2-я } \\
\text { крутильная }\end{array}$ & & 3548 & & 3505,2 & $-1,23$ \\
\hline 5-я изгибная & & 4695 & & 4890,0 & 4,16 \\
\hline $\begin{array}{l}\text { 3-я } \\
\text { крутильная }\end{array}$ & & 5360 & & 5295,9 & $-1,37$ \\
\hline
\end{tabular}

\section{Методика и результаты расчетного модального анализа}

Для расчетного модального анализа использовался метод конечных элементов (МКЭ). При пренебрежении демпфированием собственные колебания конечно-элементной модели с $n$ степенями свободы описываются в матричной форме уравнением $[7,26]$

$$
[M]\{\bar{u}\}+[K]\{u\}=0,
$$

где $[K]$ и $[M]-$ матрицы жесткости и масс; $\{u\}$ и $\{\bar{u}\}$ - векторы перемещений и ускорений в узлах конечно-элементной (КЭ) модели.

Это уравнение имеет вещественное периодическое решение вида $\{u\}=\left\{u_{0}\right\} \cos \omega t$, если выполняется условие

$$
\left([K]-\omega^{2}[M]\right)\left\{u_{0}\right\}=0 .
$$

Задача расчета собственных частот и форм колебаний сводится к задаче о собственных значениях $\omega_{k}$ и векторах $\left\{u_{0}\right\}_{k}$, которые обращают в ноль определитель:

$$
\operatorname{det}\left|[K]-\omega^{2}[M]\right|=0 .
$$


Поставленная задача решалась методом конечных элементов в пакете ANSYS Workbench. Принималось, что образец-пластина свободна от ограничений на перемещения («свободный подвес»). Конечно-элементная модель состоит из восьмиузловых призматических элементов (рис. 4), при этом в рамках од-

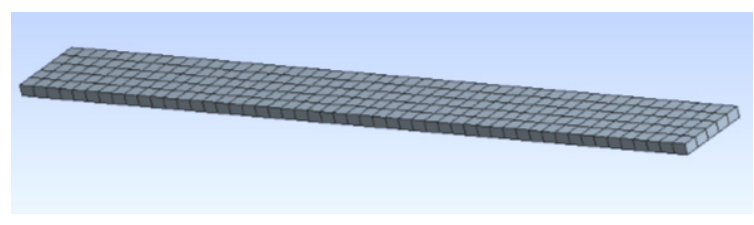

Рис. 4. Конечно-элементная модель образца ного элемента объединяются все 20 слоев углепластика, ориентированных различным образом. Характерный размер элементов в плоскости пластины выбирался на основе оценки сходимости результатов расчета собственных частот. Серия расчетов с размерами элементов от 5 до 1,25 мм показала, что при размере элемента 2,5 мм (около 1000 элементов) имеет место сходимость значений первых восьми собственных частот с погрешностью в пределах $0,3 \%$.

Модель материала образца - слоистый композит с ортотропными линейно упругими слоями. Принималось, что различно ориентированные слои композита деформируются совместно без проскальзывания. Технические упругие постоянные углепластика принимались как по результатам испытаний стандартных образцов (для $E_{11}, E_{22}, G_{12}$ ), так и по оценочным данным из работ [3, 25, 27] (табл. 3).

Таблица 3

Характеристики* материала ВКУ-39

\begin{tabular}{|c|c|c|c|c|c|c|c|c|c|c|}
\hline Источник & $\rho, \kappa г / \mathrm{M}^{3}$ & $E_{11}$, ГПа & $E_{22}$, ГПа & $E_{33}$, ГПа & $G_{12}$, ГПа & $G_{13}$, ГПа & $G_{23}$, ГПа & $v_{21}$ & $v_{13}$ & $v_{32}$ \\
\hline $\begin{array}{l}\text { По данным } \\
{[3,23,25]}\end{array}$ & 1580 & 63,9 & 63,9 & 20 & 4,8 & 2,7 & 2,7 & 0,04 & 0,3 & 0,3 \\
\hline $\begin{array}{l}\text { После иден- } \\
\text { тификации }\end{array}$ & 1580 & 70,2 & 67,4 & 18,3 & 4,2 & 2,9 & 2,6 & 0,04 & 0,3 & 0,3 \\
\hline
\end{tabular}

* Обозначения: $E_{11}$ - модуль упругости вдоль основы; $E_{22}$ - модуль упругости поперек основы; $E_{33}$ - модуль упругости поперек плоскости слоя; $G_{12}, G_{13}, G_{23}$ - модули сдвига; $v_{21}, v_{13}, v_{32}-$ коэффициенты Пуассона, $\rho$ - плотность.

В результате расчетного модального анализа в диапазоне до 6400 Гц выявлено пять изгибных собственных форм колебаний, три крутильных, а также две изгибные в плоскости пластины. В описанном выше экспериментальном исследовании две последние вследствие причин методического характера выявлены не были. Остальные собственные формы совпали с экспериментальными (см. табл. 2). Значения соответствующих собственных частот также приведены в табл. 2. Расхождение расчетных и экспериментальных значений собственных частот лежит в пределах $7 \%$. В некоторых практически важных случаях такая точность расчета собственных частот может оказаться недостаточной для отстройки от резонансных колебаний детали.

\section{Идентификация параметров модели материала на основе результатов экспериментального модального анализа}

Цель идентификации (настройки) модели материала состояла в уточнении характеристик материала: модулей упругости вдоль основы $E_{11}$ и поперек основы $E_{22}$, модуля упругости поперек плоскости слоя $E_{33}$, модулей сдвига $G_{12}, G_{13}, G_{23}$ и коэффициентов Пуассона $v_{21}, v_{13}, v_{32}$. Для идентификации использовались результаты экспериментального модального анализа - собственные частоты и формы колебаний образца.

Задача идентификации модели материала рассматривается как задача оптимизации [28, 29] с целевой функцией: 


$$
I=\sum_{i=1}^{n} \alpha_{i}\left(\frac{f_{i p}-f_{i e}}{f_{i e}}\right)^{2} \rightarrow \min
$$

где $f_{i p}$ и $f_{i e}$ - расчетное и экспериментальное значения $i$-й собственной частоты; $\alpha_{i}-$ весовые коэффициенты.

Параметрами управления считаются характеристики материала $E_{11}, E_{22}, E_{33}, G_{12}, G_{13}, G_{23}$, $v_{21}, v_{13}, v_{32}$. В качестве ограничений на параметры управления принималось отклонение от исходного значения на $\pm 8 \%$.

В соотношении (2) важно, чтобы расчетные и экспериментальные значения собственных частот соответствовали одним и тем же собственным формам. В настоящей работе сравнение расчетных и экспериментальных собственных форм проводилось на основании анализа их анимационного представления. В более сложных случаях для этого может быть использован анализ МАС-матрицы [21].

Для решения задачи оптимизации использовался метод квазислучайного поиска. Весовые коэффициенты были приняты равными единице. Квазислучайный поиск включал в себя 100 реализаций.

В результате расчетов был получен набор параметров управления, при котором целевая функция (2) снизилась по сравнению с исходным значением в три раза. Эти значения приведены в табл. 3. Полученные для этих параметров значения собственных частот приведены в табл. 4. Расхождение с экспериментальными данными составляет 3,9 \% для крутильных форм колебаний и 2,9\% для изгибных форм, что значительно лучше, чем до идентификации.

Таблица 4

Погрешность расчета собственных частот колебаний образца

\begin{tabular}{|l|c|c|c|c|c|}
\hline \multirow{2}{*}{$\begin{array}{c}\text { № } \\
\text { п/п }\end{array}$} & \multirow{2}{*}{$\begin{array}{c}\text { Эксперимент, } \\
\text { собственная } \\
\text { частота, Гц }\end{array}$} & $\begin{array}{c}\text { Расчет МКЭ без идентификации } \\
\text { частота, Гц }\end{array}$ & $\begin{array}{c}\text { Расчет МКЭ после идентификации } \\
\text { с экспериментом, \% }\end{array}$ & $\begin{array}{c}\text { Росхожстенная } \\
\text { частота, Гц }\end{array}$ & $\begin{array}{c}\text { Расхождение } \\
\text { с экспериментом, \% }\end{array}$ \\
\hline 1-я изгибная & 363 & 389,0 & 7,05 & 374 & 2,9 \\
\hline 2-я изгибная & 997 & 1063,2 & 6,60 & 1022 & 2,4 \\
\hline 1-я крутильная & 1763 & 1744,7 & $-1,02$ & 1699 & $-3,8$ \\
\hline 3-я изгибная & 1940 & 2057,4 & 6,01 & 1980 & 2,0 \\
\hline 4-я изгибная & 3180 & 3342,9 & 5,14 & 3220 & 1,2 \\
\hline 2-я крутильная & 3548 & 3505,2 & $-1,23$ & 3414 & $-3,9$ \\
\hline 5-я изгибная & 4695 & 4890,0 & 4,16 & 4717 & 0,5 \\
\hline 3-я крутильная & 5360 & 5295,9 & $-1,37$ & 5158 & $-3,9$ \\
\hline
\end{tabular}

На рис. 5 приведена диаграмма, демонстрирующая чувствительность четырех низших собственных частот колебаний к параметрам материала. Видно, что чувствительность собственных частот, а значит, и целевой функции к параметрам материала неодинакова. Наибольшее влияние на все собственные частоты колебаний оказывает модуль упругости в направлении основы $E_{11}$, существенно меньшее - $E_{22}$. К модулям сдвига целевая функция чувствительна слабо. Влияние модулей сдвига и модуля упругости в направлении утка на крутильную форму колебаний несколько больше, чем на изгибные формы.

Этот вывод можно использовать для уменьшения трудоемкости расчетов по идентификации моделей подобных материалов. 


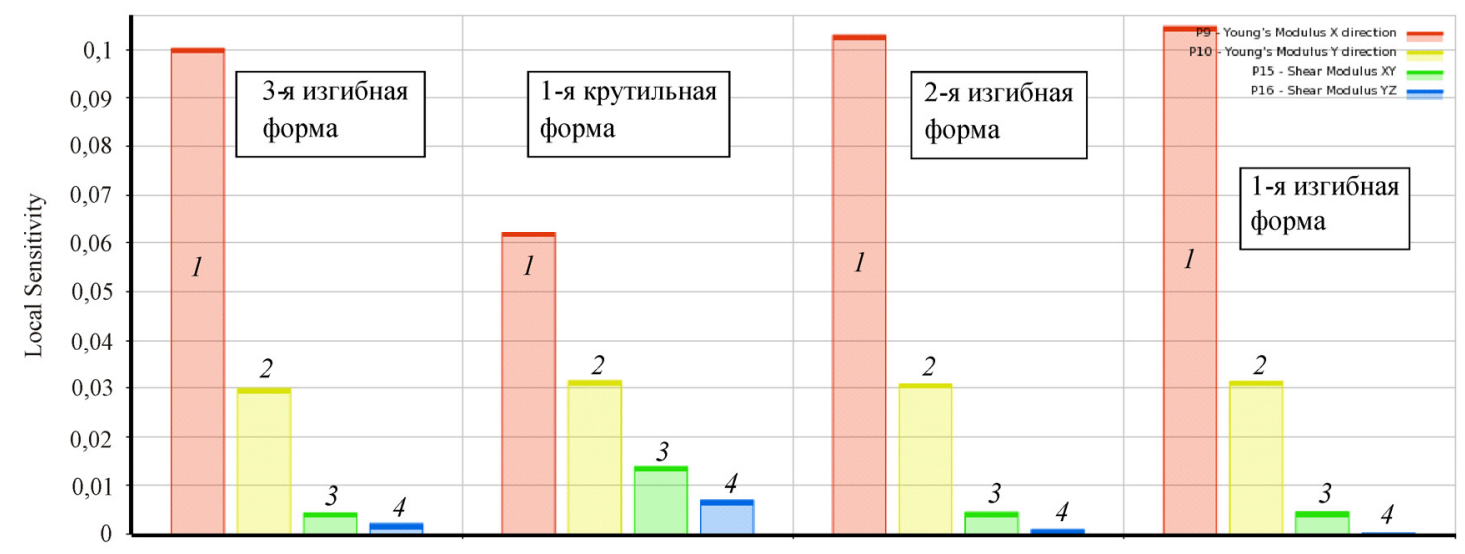

Рис. 5. Оценка чувствительности расчетных собственных частот к параметрам материала:

$$
1-E_{11} ; 2-E_{22} ; 3-G_{12} ; 4-G_{23}
$$

\section{Заключение}

В результате проведенного исследования отработана методика идентификации параметров модели упругого поведения слоистых ПКМ на основании экспериментальных данных о модальных характеристиках (собственных частотах и формах колебаний) образцов. Основные элементы методики сводятся к следующему.

В качестве образца для исследований используется рекомендованная стандартами ASTM для испытаний на растяжение и усталость пластина прямоугольной формы. Образец крепится в податливых эластичных подвесах таким образом, чтобы условия закрепления - отсутствие ограничений на перемещения - были максимально приближены к реализуемым в расчетах.

Для проведения экспериментального модального анализа рекомендуется трехкомпонентная сканирующая лазерная виброметрия, с помощью которой настоящей работе удалось получить собственные частоты колебаний образца из ПКМ в диапазоне частот до 6400 Гц с весьма незначительным рассеянием (в пределах 0,5\%), а собственные формы - с высоким пространственным разрешением.

Используемая для идентификации конечно-элементная модель образца должна обеспечивать сходимость результатов расчета собственных частот также в пределах 0,5 \%. Для решения задачи оптимизации может быть эффективно использован метод квазислучайного поиска. В качестве параметров управления для слоистых ПКМ на тканой основе можно ограничиться модулями упругости $E_{11}$ и $E_{22}$.

Разработанная методика может быть рекомендована для определения параметров модели материала, необходимых для расчетов модальных характеристик изделий из ПКМ.

\section{Библиографический список}

1. Иноземцев А.А., Нихамкин М.Ш., Сандрацкий В.Л. Основы конструирования авиационных двигателей и энергетических установок. - М., Машиностроение, 2008. - Т. 2. - 368 с. - (Газотурбинные двигатели).

2. Савин С.П. Применение современных полимерных композиционных материалов в конструкции планера самолетов семейства МС-21 // Известия Самар. науч. центра РАН. - 2012. - Т. 14, № 4(2). C. 686-693.

3. Технологии и задачи механики композиционных материалов для создания лопатки спрямляющего аппарата авиационного двигателя / А.Н. Аношкин, В.Ю. Зуйко, Г.С. Шипунов, А.А. Третьяков // Вестник Пермского национального исследовательского политехнического университета. Механика. 2014. - № 4. - C. 5-44. 
4. Келли А. Инженерный триумф углеволокон // Композиты и наноструктуры. - 2009. - № 1. C. 38-49.

5. Стреляев Д.В., Умушкин Б.П., Никонов В.В. Перспективные композиционные материалы в конструкциях авиационной и космической техники / Моск. гос. техн. ун-т гражд. авиации. - М., 2012. - 73 с.

6. Скворцов Ю.В. Конспект лекций по дисциплине механика композиционных материалов / Самар. гос. аэрокосм. ун-т. - Самара, 2013. - 94 с.

7. Леонтьев Н.В. Применение системы ANSYS к решению задач модального и гармонического анализа / Нижегород. гос. ун-т им. Н.И. Лобачевского. - Н. Новгород, 2006. - 101 с.

8. Расчетно-экспериментальные исследования собственных частот и форм колебаний лопатки спрямляющего аппарата из полимерных композиционных материалов / М.А. Гринев, А.Н. Аношкин, П.В. Писарев, Г.С. Шипунов, М.Ш. Нихамкин, А.А. Балакирев, И.П. Конев, А.А. Головкин // Вестник Пермского национального исследовательского политехнического университета. Механика. - 2016. - № 4. C. 106-119.

9. Нихамкин М.Ш., Семенова И.В. Концентрация напряжений в лопатках компрессора при повреждении их посторонними предметами // Известия вузов. Авиационная техника. - 2011. - № 4. - С. 15-18.

10. Ефимик В.А. Применение метода конечных элементов к задаче обственных колебаний прямоугольных пластин и цилиндрических оболочек // Вестник Пермского национального исследовательского политехнического университета. Аэрокосмическая техника. - 2014. - № 38. - С. 72-92.

11. Mistuning identification and model updating of an industrial blisk / D. Laxalde, F. Thouverez, J.-J. Sinou, J.-P. Lombard, S. Baumhauer // International Journal of Rotating Machinery. - Vol. 2007. - Article ID $17289 .-10$ p. DOI: $10.1155 / 2007 / 17289$

12. Ткач В.В. Применение модального анализа в многодисциплинарном исследовании ЖРД // Электронный журнал «Труды МАИ». - 2010. - № 38.

13. Безмозгий И.М., Софинский А.Н., Чернягин А.Г. Моделирование в задачах вибропрочности конструкций ракетно-космической техники // Космическая техника и технологии. - 2014. - № 3(6). C. 71-80.

14. Межин В.С., Обухов В.В. Практика применения модальных испытаний для целей верификации конечно-элементных моделей конструкции изделий ракетно-космической техники // Космическая техника и технологии. - 2014. - № 1(4). - С. 86-91.

15. Методика уточнения конечно-элементной модели механической системы с помощью анализа чувствительности / С.М. Николаев, В.А. Жулёв, И.А. Киселёв, П.С. Воронов // Наука и образование. Электрон. журн. МГТУ им. Н.Э. Баумана. - 2014. - № 12. - С. 128-136.

16. Николаев С. М. Жулёв В. А. Киселёв И. А. Уточнение конечно-элементной модели лопатки ГТД на основе результатов вибрационных испытаний с учетом разброса модальных параметров // Наука и образование. Электрон. журнал. МГТУ им. Н.Э. Баумана. - 2015. - № 09. - С. 336-351.

17. Калиненкова А.О. Исследование свойств сотового заполнителя по результатам динамических испытаний // Молодеж. науч.-техн. сб.: электрон. журнал. - 2015. - № 12.

18. ASTM D 3039/D 3039M-14. Standard Test Method for Tensile Properties of Polymer Matrix Composite Materials / ASTM International. - West Conshohocken, PA, 19428-2959 USA, 2014.

19. ASTM D 3479. Standard Test Method for Tension-Tension Fatigue of Polymer Matrix Composite Materials / ASTM International. - West Conshohocken, PA, 19428-2959 USA, 2012.

20. Ewins D.J. Modal testing: Theory, practice and application. - 2nd ed. - Baldock: Research Studies Press LTD, 2000.

21. Heylen W., Lamens S., Sas P. Modal analyses. Theory and testing / Leven. University. Leuven, 2003. $-325 \mathrm{p}$.

22. Методика экспериментального модального анализа лопаток и рабочих колес газотурбинных двигателей / А.А. Иноземцев, М.Ш. Нихамкин, Л.В. Воронов, А.Б. Сенкевич, А.Ю. Головкин, Б.П. Болотов // Тяжелое машиностроение. - 2010. - № 11. - С. 2-6.

23. Вибрационные измерения в аэрокосмической отрасли [Электронный ресурс] / Advancing Measurements by Ligh. Polytec. - URL: www.polytec.com (дата обращения: 20.09.2017).

24. Экспериментальный и расчетный модальный анализ лопаток вентиляторов полой конструкции / А.А. Иноземцев, М.Ш. Нихамкин, Л.В. Воронов, И.Л. Гладкий, А.Ю. Головкин, Б.П. Болотов // Авиационная промышленность. - 2010. - № 3. - С. 8-11. 
25. Компьютерное моделирование механического поведения композитной лопатки спрямляющего аппарата авиационного двигателя / М.А. Гринев, А.Н. Аношкин, П.В. Писарев, В.Ю. Зуйко, Г.С. Шипунов // Вестник Пермского национального исследовательского политехнического университета. Механика. - 2015. - № 3. - С. 38-51.

26. Зенкевич О. Метод конечных элементов в технике. - М.: Мир. -542 с.

27. Исследование НДС и оценка прочности композитной лопатки спрямляющего аппарата авиационного двигателя / М.А. Гринёв, А.Н. Аношкин, П.В. Писарев, В.Ю. Зуйко, Г.С. Шипунов // Вестник Пермского национального исследовательского политехнического университета. Механика. - 2015. - № 4. С. 293-307.

28. Колмогоров Г.Л., Лежнева А.А. Оптимальное проектирование конструкций: учеб. пособие / Перм. гос. техн. ун-т. - Пермь, 2005. - 168 с.

29. Алексеева Е.В., Кутненко О.А., Плясунов А.В. Численные методы оптимизации: учеб. пособие / Новосиб. ун-т. - Новосибирск, 2008. - 128 с

\section{References}

1. Inozemtsev A.A., Nikhamkin M.Sh., Sandratskiy V.L. Osnovy konstruirovaniya aviatsionnykh dvigateley i energeticheskikh ustanovok [Basics of designing aircraft engines and power plants]. Seriya: Gazoturbinnye dvigateli t. 2. M., Mashinostroenie, 2008, 368 p.

2. Savin S.P. Primenenie sovremennykh polimernykh kompozitsionnykh materialov v konstruktsii planera samoletov semeystva MS-21 [The use of modern polymer composite materials in the design of the airframe of the MS-21 family aircraft]. Izvestiya Samarskogo nauchnogo tsentra Rossiyskoy akademii nauk, 2012 , Vol. 14, no. 4(2), pp. 686-693.

3. Anoshkin A.N., Zuyko V.Yu., Shipunov G.S., Tretyakov A.A. Tekhnologii i zadachi mekhaniki kompozitsionnykh materialov dlya sozdaniya lopatki spryamlyayushchego apparata aviatsionnogo dvigatelya [Technologies and tasks of the mechanics of composite materials for the creation of a blade of a rectifying device for an aircraft engine]. Vestnik PNIPU. Mekhanika, 2014, no. 4, pp. 5-44.

4. Kelli A. Inzhenernyy triumf uglevolokon. Kompozity i nanostruktury [Engineering triumph of carbon fiber. Composites and nanostructures.]. 2009, no. 1, pp. 38-49.

5. Strelyaev D.V., Umushkin B.P., Nikonov V.V. Perspektivnye kompozitsionnye materialy v konstruktsiyakh aviatsionnoy i kosmicheskoy tekhniki [Perspective composite materials in the constructions of aviation and space technology]. Moscow, MGTU GA, 2012, 73 p.

6. Skvortsov Yu.V. Konspekt lektsiy po distsipline mekhanika kompozitsionnykh materialov [Lecture notes on the discipline of the mechanics of composite materials]. Samara. SGAU, 2013, $94 \mathrm{p}$.

7. Leontev N.V. Primenenie sistemy ANSYS k resheniyu zadach modalogo i garmonicheskogo analiza [Application of the ANSYS system to solving modal and harmonic analysis problems]. Nizhniy Novgorod, $N N G U, 2006,101 \mathrm{p}$.

8. Grinev M.A., Anoshkin A.N., Pisarev P.V., Shipunov G.S., Nikhamkin M.Sh, Balakirev A.A., Konev I.P., Golovkin A.A. Raschetno-eksperimentalnye issledovaniya sobstvennykh chastot i form kolebaniy lopatki spryamlyayushchego apparata iz polimernykh kompozitsionnykh materialov [Calculation and experimental studies of the natural frequencies and vibration modes of the blade of a rectifying apparatus made from polymer composite materials]. Vestnik PNRPU. Mekhanika, 2016, no. 4, pp. 106-119.

9. Nikhamkin M.Sh., Semenova I.V. Kontsentratsiya napryazheniy v lopatkakh kompressora pri povrezhdenii ikh postoronnimi predmetami [Concentration of stresses in the compressor blades if they are damaged by foreign objects]. Izvestiya vysshikh uchebnykh zavedeniy. Aviatsionnaya tekhnika, 2011, no. 4, pp. 15-18.

10. Efimik V.A. Primenenie metoda konechnykh elementov k zadache sobstvennykh kolebaniy pryamougolnykh plastin i tsilindricheskikh obolochek [The application of the finite element method to the problem of general oscillations of rectangular plates and cylindrical shells]. Vestnik PNRPU. Aerokosmicheskaya tekhnika, 2014, no. 38, pp. 72-92.

11. Laxalde D., Thouverez F., Sinou J.-J., Lombard J.-P., Baumhauer S. Mistuning identification and model updating of an industrial blisk. International Journal of Rotating Machinery, vol. 2007, Article ID 17289, 10 p. DOI: $10.1155 / 2007 / 17289$

12. Tkach V.V. Primenenie modalnogo analiza v mnogodistsiplinarnom issledovanii ZhRD [The use of modal analysis in a multidisciplinary study]. Elektronnyy zhurnal «Trudy MAI», 2010, no. 38. 
13. Bezmozgiy I.M., Sofinskiy A.N., Chernyagin A.G. Modelirovanie v zadachakh vibroprochnosti konstruktsiy raketno-kosmicheskoy tekhniki [Modeling in the problems of vibration resistance of rocket and space engineering structures]. Kosmicheskaya tekhnika i tekhnologii, 2014, no. 3 (6)/2014, pp.71-80.

14. Mezhin V.S., Obukhov V.V. Praktika primeneniya modalnykh ispytaniy dlya tseley verifikatsii konechno-elementnykh modeley konstruktsii izdeliy raketno-kosmicheskoy tekhniki [The practice of applying modal tests for the purposes of verification of finite element models of the design of rocket and space equipment]. Kosmicheskaya tekhnika i tekhnologii, 2014, no. 1(4)/2014, pp. 86-91.

15. Nikolaev S.M., Zhulev V.A., Kiselev I.A., Voronov P.S. Metodika utochneniya konechnoelementnoy modeli mekhanicheskoy sistemy s pomoshchyu analiza chuvstvitelnosti. [Method for refining the finite element model of a mechanical system using sensitivity analysis]. Nauka i Obrazovanie. MGTU im. N.E. Baumana. Elektron. Zhurn, 2014, no. 12, pp. 128-136.

16. Nikolaev S.M., Zhulev V.A., Kiselev I.A. Utochnenie konechno-elementnoy modeli lopatki GTD na osnove rezul'tatov vibratsionnykh ispytaniy s uchetom razbrosa modalnykh parametrov [Refinement of the finite-element model of the GTE blade based on the results of vibration tests taking into account the spread of the modal parameters]. Nauka i Obrazovanie. MGTU im. N.E. Baumana. Elektron. Zhurn, 2015, no. 09, pp. 336-351.

17. Kalinenkova A.O. Issledovanie svoystv sotovogo zapolnitelya po rezultatam dinamicheskikh ispytaniy [Investigation of properties of honeycomb core by results of dynamic tests]. Molodezhnyy nauchnotekhnicheskiy sbornik. Elektronnyy zhurnal, 2015, no. 12.

18. ASTM D 3039/D 3039M - 14. Standard Test Method for Tensile Properties of Polymer Matrix Composite Materials. ASTM International, West Conshohocken, PA, 19428-2959 USA, 2014.

19. ASTM D 3479. Standard Test Method for Tension-Tension Fatigue of Polymer Matrix Composite Materials. ASTM International, West Conshohocken, PA, 19428-2959 USA, 2012.

20. Ewins D.J. Modal Testing: Theory, Practice and Application// 2nd edition. Baldock, Research Studies Press LTD, 2000.

21. Heylen W., Lamens S., Sas P. Modal analyses. Theory and testing. Leven Univ. Belgium, 2003, $325 \mathrm{p}$.

22. Inozemtsev A.A., Nikhamkin M.Sh., Voronov L.V., Senkevich A.B., Golovkin A.Yu., Bolotov B.P. Metodika eksperimentalnogo modalnogo analiza lopatok i rabochikh koles gazoturbinnykh dvigateley [Technique of experimental modal analysis of blades and impellers of gas turbine engines]. Tyazheloe mashinostroenie, 2010, no. 11, pp. 2-6.

23. Vibratsionnye izmereniya $\mathrm{v}$ aerokosmicheskoy otrasli [Vibration measurements of the aerospace industry]. Advancing Measurements by Ligh. Polytec, available at: www.polytec.com (accessed 20 September 2017).

24. Inozemtsev A.A., Nikhamkin M.Sh., Voronov L.V., Gladkiy I.L., Golovkin A.Yu., Bolotov B.P. Eksperimentalnyy i raschetnyy modalnyy analiz lopatok ventilyatorov poloy konstruktsii [Experimental and calculated modal analysis of fan blades of hollow structure]. Aviatsionnaya promyshlennost, 2010, no. 3, pp. 8-11.

25. Grinev M.A., Anoshkin A.N., Pisarev P.V., Zuyko V.Yu., Shipunov G.S. Kompyuternoe modelirovanie mekhanicheskogo povedeniya kompozitnoy lopatki spryamlyayushchego apparata aviatsionnogo dvigatelya [Computer modeling of the mechanical behavior of a composite blade of an aircraft engine straightener]. Vestnik PNIPU. Mekhanika, 2015, no. 3, pp. 38-51.

26. Zenkevich O. Metod konechnykh elementov $\mathrm{v}$ tekhnike [Finite element method in engineering]. Moscow: Mir, $542 \mathrm{p}$.

27. Grinev M.A., Anoshkin A.N., Pisarev P.V., Zuyko V.Yu., Shipunov G.S. Issledovanie NDS i otsenka prochnosti kompozitnoy lopatki spryamlyayushchego apparata aviatsionnogo dvigatelya[Research of VAT and an estimation of durability of a composite blade of rectifying device of the aviation engine]. Vestnik PNIPU. Mekhanika, 2015, no. 4, pp. 293-307.

28. Kolmogorov G.L., Lezhneva A.A. Optimalnoe proektirovanie konstruktsiy [Optimal design of structures: Textbook]. PGTU. Perm, 2005, 168 p.

29. Alekseeva E.V., Kutnenko O.A., Plyasunov A.V. Chislennye metody optimizatsii [Numerical optimization methods: Textbook]. Novosib. un-t. Novosibirsk, 2008, 128 p. 


\section{Сведения об авторах}

Нихамкин Михаил Шмерович (Пермь, Россия) - доктор технических наук, профессор кафедры «Авиационные двигатели» ФГБОУ ВО ПНИПУ (614990, Пермь, Комсомольский пр., д. 29, е-mail: nikhamkin@mail.ru).

Соломонов Данил Глебович - студент ФГБОУ ВО ПНИПУ (614990, Пермь, Комсомольский пр., д. 29, e-mail: solomonov1198@yandex.ru).

\section{About the authors}

Mikhail Sh. Nikhamkin (Perm, Russian Federation) - Doctor of Technical Sciences, Professor, Department of Aircraft Engines, Perm National Research Polytechnic University (29, Komsomolsky av., Perm, 614990, Russian Federation, e-mail: nikhamkin@mail.ru).

Danil G. Solomonov (Perm, Russian Federation) - Student, Perm National Research Polytechnic University (29, Komsomolsky av., Perm, 614990, Russian Federation, e-mail: solomonov1198@yandex.ru).

Получено 18.11.2017 